\title{
Articles
}

\author{
EMMA GÓMEZ NICOLAU ${ }^{1}$ \\ LAURA CASTILLO MATEU²
}

\section{Diversiones: producciones y prácticas de género ${ }^{3}$}

\section{Diversions: Productions and Practices of Gender}

\begin{abstract}
RESUMEN
El objetivo del presente artículo es enmarcar las aportaciones al monográfico «Diversiones. Producciones y prácticas de género» en algunas teorías que permitan entender el juego entre estructura y agencia a la hora de abordar las prácticas y producciones de género. Para ello, se introduce la teoría del orden de género de Raewyn Connell $(2006,2009,2018)$ y la teoría de la performatividad de Judith Butler $(2002,2007,2009)$, de manera que los contenidos de la revista se puedan leer, bien desde el análisis crítico de alguna de las dimensiones de la estructura de género, bien desde los agenciamientos que permiten tanto las prácticas encarnadas de género como los activismos que devienen resistencias y subversiones. Prácticas y producciones que contienen lo diverso, las versiones y la diversión.
\end{abstract}

Palabras clave: relaciones de género, estructura, agencia, feminismos, activismos.

\section{Abstract}

The main goal of the article is to set a framework to the contributions of the present volume «Diversions: productions and practices of gender» from some theories that allow to understand the game between structure and agency to tackle the productions and practices of gender. To get it, we introduce Raewyn Connell's gender order theory $(2006,2009,2018)$ and Judith Butler's performativity theory $(2002,2007,2009)$, so the contributions can be read, either from the critical analysis of any of the dimensions of gender structure, either from the agency displayed both, embodied practices of gender and activisms, that become resistances and subversions. That means productions and practices that contain the diverse, the versions and the diversion.

Keywords: gender relations, structure, agency, feminisms, activisms.

\section{Sumario}

1. Introducción. 2. Estructura, agencia, memoria y transformación social. 3. Diversiones: diálogos entre la academia y el activismo. 4. Por una política de la diversión. Bibliografía.

1 Universitat Jaume I, Departamento de Filosofía y Sociología, enicolau@uji.es.

2 Universitat Jaume I, Departamento de Ciencias de la Comunicación, lcastill@uji.es.

3 Emma Gómez Nicolau y Laura Castillo Mateu investigan en el marco del proyecto «La re-significación de la mujer-víctima en la cultura popular: implicaciones para la innovación representacional en la construcción de la vulnerabilidad y la resistencia» (FEM2015-65834-C2-2-P). 


\section{Introducción}

Diversiones contiene lo diverso, las versiones y la diversión. La voluntad de aunar estos significantes en el título que abre este número de Asparkía. Investigació feminista se sustenta en el abordaje del género desde una perspectiva que permita vislumbrar tanto los constreñimientos del género, que devienen desigualdades, jerarquías y precariedades, como las posibilidades diversas y, en este sentido, también radicales, marginales, imaginativas y creativas de encarnar el género.

No es fácil encontrar una propuesta sobre género que consiga equilibrar en el análisis el peso de la estructura social y, al mismo tiempo, las posibilidades de transgredir, de inventar y, por supuesto de alterar el orden social. La contraposición entre estructura y agencia ha sido unos de los espacios más fructíferos en la exploración teórica del género y también de la práctica activista feminista. En este artículo iniciamos, en primer lugar, un pequeño recorrido por algunas de las articulaciones teóricas que nos permiten acercarnos a ese nudo gordiano que es la concepción del género como algo relacional, procesual e histórico, en cuyo proceso de articulación encontramos las tensiones entre la contingencia y la agencia.

En segundo lugar, ponemos a dialogar con este pequeño entramado teórico los contenidos de Diversiones ya que, todas sus secciones-Articles, Retrat, Textos, Creació literaria, Llibres y Il-lustracions- indagan en alguna de las dimensiones de la estructura de las relaciones de género y/o en las prácticas políticas que desafían o subvierten el orden de género. Por último, nos atrevemos a proponer una política de la diversión, reivindicando las dimensiones del goce, el placer y los cuidados como espacios en los que es posible resignificar el género y subvertir en el marco de los movimientos sociales y los activismos.

\section{Estructura, agencia, memoria y transformación social}

La teorización sobre género es una constante desde los años 70 del siglo XX, a pesar de lo cual todavía nos encontramos, en algunas ocasiones, una sub-teorización del mismo en los abordajes de muchos artículos e investigaciones. Pareciera que el concepto género se da por supuesto, sin atender a su profundidad y a la diversidad de perspectivas teóricas que contiene. No es este el lugar para extendernos en este tema, pero queremos aprovechar para recorrer algunas teorizaciones que abordan el debate sobre la agencia, y no solo por poner en evidencia el valor de cambio social de la capacidad de transgredir las normas sociales, sino por remarcar que la realidad social es mucho menos dicotómica que lo que le es funcional al sistema heteropatriarcal y ha venido siendo explicativo para algunas elaboraciones teóricas hegemónicas que perpetúan la dicotomía sobre un rígido esquema sexo/género -que si bien ha superado el biologicismo, recrea a menudo una jaula cultural que oprime las realizaciones de género tanto o más que lo haría la biología, situando al patriarcado como un sistema de organización social ahistórico (Osborne y Molina, 2008; Connell, 2009). Tal y como exponen Osborne y Molina en referencia a las aportaciones de Gayle Rubin, «toda sociedad tiene modos sistemáticos de lidiar 
con el sexo y el género. Pero es importante distinguir entre la capacidad y necesidad humanas de crear un mundo sexuado y las formas empíricamente opresivas en que los mundos sexuales han sido organizados»(Osborne y Molina, 2008: 152). Esta hermosa cita nos abre el camino para vislumbrar cómo el mundo sexuado puede organizarse de manera ética, no opresiva y no jerárquica. No obstante, hay miradas que nos llevan más allá.

La mirada constructivista del género de Judith Butler $(2002,2007)$ evidenciará la discontinuidad radical entre cuerpos sexuados y géneros socialmente construidos y desestabilizará la relación mimética entre género y sexo. Refuta el carácter invariable del sexo al afirmar que «quizás esta construcción denominada sexo esté tan culturalmente construido como el género; de hecho, quizás siempre fue género, con el resultado de que la distinción entre sexo y género no existe como tal» (Butler, 2007: 55), quedaría situado como realidad pre-discursiva en un ejercicio de construcción del sexo como algo natural, dentro de la lógica binaria naturaleza/ cultura a través de su teoría de la performatividad (Butler, 2009).

El género no designa un ser sustantivo, sino un punto de unión relativa entre conjuntos de relaciones culturales e históricas específicas en las que el cuerpo y el deseo también serán fruto de las tecnologías disciplinarias del sexo, el género y la sexualidad. Poner al descubierto las políticas reguladoras de los cuerpos, las identidades y los deseos no es únicamente un ejercicio intelectual de aplicación de las teorías foucaultianas sobre el poder y el saber (Foucault, 1983, 1978, 1978b), sino también una expresión clara y deliberada de las posibilidades de resistencia (donde hay poder, hay resistencia) contra la violencia aplicada a las sexualidades no normativas, a las identidades múltiples y a las encarnaciones divergentes del género. Subvertir el orden de género - propuesta que entronca con la de la abolición de las categorías de hombre y mujer de Wittig (2006)-, deviene un ejercicio de desenmascaramiento de las «verdades» opresivas.

Las nociones de "contingencia» $\mathrm{y}$ «agencia» le permiten a Butler huir tanto de los límites del determinismo de género, como de los enfoques basados en la libertad de creación absoluta: el falogocentrismo y los instrumentos disciplinarios de los géneros marcan unas posiciones identitarias, sexuales y físicas que serán imposibles de alcanzar completamente por parte de los cuerpos localizados. Desde las posiciones localizadas se producirán formas genéricas diversas, algunas de las cuales tienen la capacidad de subvertir el género -cuestionarlo, invertirlo, desnaturalizarlo.

Gays, lesbianas, identidades butch/femme, drag queens, drag kings, intersexuales, transexuales y otras identidades evidenciarán la absoluta disociación entre sexo, identidad y sexualidad y, además, pondrán de manifiesto la identidad de género como una ficción reguladora infringida de manera violenta. La hiperfeminización teatralizada en drag queens (y la hipermasculinización teatralizada en drag kings) no son más que la imitación de identidades de género que no tienen una base real sino que funcionan como un ideal ficticio, la imitación de una quimera. Quimera que, sin embargo, tiene una base material y estructurante de las relaciones sociales. 
Esta base material, y esas formas empíricamente opresivas a las que se refería Rubin, parten de las relaciones sociales que se establecen en un contexto determinado, las formas en las que las personas, los grupos y las organizaciones están conectadas y divididas (Connell, 2009; Connell y Pearse, 2018). Relaciones de género que se hacen y se rehacen continuamente, y en cuyo marco cada individuo hace su propio género, aunque no de manera libre, sino sujetado, fuertemente influenciado por el orden de género en el que nos encontramos. Según la teoría de Raewyn Connell, las disposiciones de género de una sociedad son una estructura social en sí mismas que definen las posibilidades de acción, y establece cuatro dimensiones en la estructura de las relaciones de género: poder, producción, cathexis y simbolismo.

El poder incluye tanto el poder directo y coactivo que ejercen varones sobre mujeres -poder patriarcal- que se ejerce a través de los Estados, la burocracia, la violencia marital, etc., como el poder discursivo que opera de manera íntima y difusa construyendo identidades y prácticas -como es en el caso de los discursos sobre la belleza y la moda- y el poder colonial que pulverizó las relaciones preexistentes para imponer las coloniales. Este poder, prosiguen las autoras, se disputa y ha desencadenado fórmulas diversas de protesta y contestación.

La segunda dimensión se refiere a la producción, el consumo y la acumulación generizada, que parte de la existencia fehaciente de la división sexual del trabajo -que feminiza y masculiniza las tareas, aunque esto sea cambiante en los diversos contextos sociales- y la división sexual del trabajo remunerado -empleo- y el trabajo no remunerado -de cuidados y afectivo- bajo el capitalismo moderno. El uso generizado de la fuerza de trabajo - que segrega horizontal y verticalmente, y reserva para las mujeres los trabajos peor pagados, temporales y en peores condiciones- se traduce en una distribución de beneficios también desigual.

La tercera dimensión que establece Connell se refiere a la estructuración social de las relaciones emocionales, los vínculos y los compromisos, que pueden ser positivos o negativos hacia el objeto -como la misoginia o la homofobia-o ambivalentes -tanto afectuosos como hostiles a la vez. La sexualidad, como principal escenario del vínculo emocional, aunque no se reduzca al género, se organiza fundamentalmente en base al género y asume que, en el Norte global, la atracción sexual se da entre géneros y se espera que los hogares se formen básicamente en torno al amor romántico y sean las mujeres las que se ocupen de la carga afectiva con respecto a los hijos e hijas -y la distribución desigual del amor en términos de Jónasdóttir (1993).

Por último, la dimensión del simbolismo, la cultura y el discurso, que nos sitúa en cómo las prácticas sociales se impregnan de una interpretación del mundo y los sistemas culturales reflejan intereses sociales particulares. En el Norte global, muchos estudios se han centrado en el estudio de las representaciones culturales de género, las actitudes generizadas, los sistemas de valores y sus problemas relacionados a partir de la teoría lacaniana del lenguaje como «falocéntrico», un sistema en el que el lugar de la autoridad, la subjetividad privilegiada, es siempre la masculina (Connell y Pearse, 2018: 145-167).

El estudio de estas cuatro dimensiones en la estructura de las relaciones de género nos permite analizar cómo se organiza el género en cada lugar, en cada zona 
geográfica. Existen -dirá Linda McDowell- "múltiples formas de "crear el género", de ser hombre y mujer. Tantas y tan opuestas como las versiones hegemónicas de la feminidad y la masculinidad. Tienen su especificidad geográfica e histórica, y varían en un amplio abanico de escalas espaciales» (McDowell, 2000: 41). El orden de género dominante en una sociedad varía en los contextos sociales, el proceso de construcción y reconstrucción del género, entretejido con el proceso de construcción de la raza, la clase y la dinámica del capitalismo global (Connell, 2009; Davis, 1981; Mohanty, 1988), da lugar a órdenes de género diversos en comunidades, clases sociales, entornos institucionales, etc.

Pierre Bourdieu (2000) estableció la necesidad del estudio de los procesos históricos para entender los mecanismos de reproducción del orden de género en un contexto social dado. El análisis de las fórmulas de opresión como producto histórico y la recuperación de la memoria se pueden convertir, así pues, en una herramienta para descubrir los mecanismos de reproducción de las desigualdades, de esas formas empíricamente opresivas, aunque también una estrategia para estudiar los mecanismos mediante los que el orden de género se transforma y subvierte, en una organización, institución o comunidad específica, y la manera en que estos cambios se contagian a otras esferas, organizaciones, instituciones o comunidades.

El peso de los movimientos sociales, especialmente de los activismos feministas, LGTB y queer, ha sido y sigue siendo clave para denunciar las injusticias del orden de género e impulsar cambios, y también para resistir, subvertir e inventar encarnaciones y prácticas que desafían el orden de género. «El concepto de agenciamiento -dirá Villaplana- desafía la hegemonía de lo normativo, homogéneo y fijo para hacer funcionar distintos nodos/agentes heterogéneos que se relacionan entre sí y hacia afuera» (Villaplana, 2017: 19). El agenciamiento constituye una práctica política y social que irrumpe y altera las formas dominantes de género; evidencia los juegos de poder, la distribución desigual de las precariedades, las represiones afectivas y el legado simbólico opresivo. Si bien en términos de Butler la agencia es una necesidad de concreción del propio self y, por tanto, cada encarnación del género implica agenciamiento que desafiará en mayor o menor manera el orden de género establecido, también la agencia se practica de manera colectiva hacia los actos de desposesión desde la crítica profunda al sujeto ilustrado (Butler y Athanasiou, 2013). Aunque las capacidades de agencia desde la primera acepción, la de la encarnación individual del género, se mantienen en tensión con la posición que se ocupa en la estructura social, desde la segunda mirada, la de la capacidad de agencia desde las precariedades y las políticas de desposesión que dejan a los sujetos sujetados a las lógicas de la precariedad, exclusión, violencia, etc., la desposesión abre vías a resistencias políticas.

\section{Diversiones: diálogos entre la academia y el activismo}

Las fronteras entre activismo y academia se nos tornan frágiles, móviles y difusas, y la propuesta de este número de Asparkía era hacer evidente esta fragilidad. Aunque los contenidos de cada uno de los apartados del número-Articles, Retrat, 
Textos, Creació literària, Llibres y Il-lustracions- tienen una naturaleza distinta, constituyen aportaciones de relevancia sobre el funcionamiento de la estructura, la agencia, el papel de la memoria y la voluntad y/o capacidad de transformación social sobre género. No obstante, por una cuestión de inteligibilidad, trataremos los contenidos por orden de aparición.

En primer lugar, Enrico Mora analiza en su artículo «La organización social y de género de la vista. Qué puedes mirar en una sauna de un gimnasio» los componentes normativos que regulan la visión y la mirada en un vestuario y una sauna de hombres de un gimnasio mixto. Desde la antropología del cuerpo y a través de una autoetnografía que aporta una reflexión metodológica de relevancia, expone magistralmente -y con humor- cómo la visión, lejos de ser un acto espontáneo, libre y reflejo, está sometida a un proceso institucionalizado de género. El estudio del manejo de la corporalidad propia y la ajena en el espacio reducido de la sauna pone de manifiesto la construcción de las corporalidades hegemónicas y disidentes, en este caso masculinas bajo el mandato heteronormativo del gimnasio. Un análisis que apunta a la estructuración social de las prácticas corporales en relación a la cathexis, al espacio que tienen el deseo, el placer, y la orientación sexual en un establecimiento regulado según mandatos heterosexuales que promueven relaciones emocionales homofóbicas.

Luego, Arantxa Grau i Muñoz profundiza en los «Placeres políticos: el activismo transfeminista en el Estado español y la re-politización de la sexualidad como estrategia de disidencia», donde aborda el transfeminsimo como activismo que reconfigura el cuerpo como arma política y se esfuerza por proponer lecturas repolitizadas del placer y el deseo. Aúna el análisis de los textos científicos y las prácticas políticas que, desde la producción científica, la creación artística y los activismos, articulan los contextos de posibilidades de transgresión del orden de género y se constituyen en prácticas de resistencia autóctonas, que juegan, provocan, dialogan y se enfrentan con un feminismo institucional anclado en una concepción ilustrada del sujeto. Los sujetos de placeres políticos son heterodoxos, irreverentes, rabiosxs... seres-cuerpos rescatados de las diásporas del feminismo que componen un entramado disidente que aspira a desestabilizar en orden de género a través de la resignificación del placer.

En «La diferencia sexual: ¿una ficción necesaria?», Ana Velasco Lázaro profundiza en los posicionamientos políticos de la teoría de la diferencia sexual propuesta por Rosi Braidotti y la teoría de la performatividad de Judith Butler. A modo de diálogo, transita por los puntos de encuentro, desencuentro y crítica de dos posiciones entre las que tiende puentes para evitar la exclusión. El análisis del concepto de diferencia sexual utilizado por el feminismo de la diferencia viene a sumar complejidad a los preceptos sobre el género que se despliegan en las aportaciones del volumen y a abordar los planteamientos del cambio social desde parámetros valorativos culturales no androcéntricos.

Tal y como afirman Connell y Pearse, "siempre que hablamos de "una mujer" o de "un hombre", ponemos en juego un tremendo sistema de concepciones, implicaciones, connotaciones y alusiones que se han ido acumulando a través de 
la historia cultural» (Connell y Pearse, 2018). En el artículo de Esther Rodríguez Ortiz «Vírgenes, madres y doncellas. Ser mujer durante el Franquismo a través del humor gráfico de La Nueva España» se aborda la representación de las mujeres bajo el Franquismo a través de uno de los periódicos del Movimiento. En este ejercicio de recopilación y análisis de la producción cultural de los chistes y el humor sobre las relaciones de género, se constata cómo el simbolismo analizado refleja intereses sociales particulares tremendamente patriarcales.

El último artículo, «La dona en la premsa tortosina en català (1900-1936)», de Carme Gombau Domingo, se preocupa por explorar los prototipos de mujer presente en la prensa comarcal de influencia ideológica en la zona de les Terres de l'Ebre durante el primer tercio del siglo XX. La autora lleva a cabo un minucioso ejercicio de lectura y análisis sobre los modelos de feminidad que retrataban los rotativos en una época en la que a duras penas se pudo implantar el sufragio femenino. El estudio viene a confirmar la norma en lo que a la representación mediática de la mujer se refiere y vemos como tanto en los periódicos más conservadores (como La Veu de Tortosa, vinculado ideológicamente a los sectores burgueses y católicos), como en los más implicados en transformación social del momento (sería el caso de Vida Tortosina, donde la autora localiza las primeras piezas firmadas por mujeres) la presencia de voces femeninas resulta anecdótica. Las referencias a ellas, además, se llevan a cabo para describir o apelar al prototipo de mujer útil a la hora de perpetuar el orden establecido o de desarrollar la revolución que se anuncia.

Elisabet Marco Arocas nos acerca a Angela Y. Davis como figura académica y activista con el retrato "Àngela Yvonne Davis: pensament encarnat sobre interseccionalitat i presons». En este retrato se profundiza en la apreciación de cómo una obra teórica y un legado vivo del activismo se interpreta a través de la experiencia encarnada de su autora. Desde la exploración perspicaz de la autobiografía de Ángela, el retrato se centra en sus análisis de la interseccionalidad de clase, género y raza -mucho antes que Crenshaw acuñara el término en 1989- y en la experiencia generizada que imprime el complejo industrial-penitenciario.

En la sección Textos proponemos la lectura del manifiesto «Un feminismo para el 99\%. Por eso las mujeres haremos huelga este año» firmado por un elenco de teóricas y activistas feministas -Linda Alcoff, Cinzia Arruzza, Tithi Bhattacharya, Rosa Clemente, Angela Davis, Zillah Eisenstein, Liza Featherstone, Nancy Fraser, Barbara Smith y Keeanga-Yamahtta Taylor- publicado originalmente en inglés en The Guardian ${ }^{4}$ el 27 de enero de 2018 y del que ofrecemos la traducción realizada por Latfem ${ }^{5}$. Este manifiesto perfila los contextos de acción del movimiento feminista estadounidense, las luchas y las principales reivindicaciones que se plasman en la práctica política de la huelga feminista del 8 de marzo de 2018. Es

4 We need a feminism for the $99 \%$. That's why women will strike this year. The Guardian. Recuperado en https://www.theguardian.com/commentisfree/2018/jan/27/we-need-a-feminism-for-the-99thats-why-women-will-strike-this-year

5 Necesitamos un feminismo para el 99\%. Por eso las mujeres haremos huelga este año. Latfem. Recuperado en http://latfem.org/necesitamos-un-feminismo-para-el-99-por-eso-las-mujeresharemos-huelga-este-ano/ 
un texto, a nuestro parecer, no solo explicativo de los modos en que el régimen de género genera desigualdad, pobreza y violencia, sino también de la capacidad de acción política y transformación social que parte de la toma de conciencia colectiva. Teniendo en cuenta el impacto de la huelga feminista y de las movilizaciones del 8 de marzo de 2018 en España, organizadas y acontecidas durante la misma edición del número, no quisimos dejar pasar la oportunidad de incorporar un texto en el que también se reivindica la potencia de fórmulas discursivas de resistencia como las campañas \#MeToo, \#UsToo y \#TimesUp, en este intento por hacer dialogar lo académico con lo activista (que hoy en día, poco se escapa a lo mediático).

En Creació literaria hemos considerado especialmente la irreverencia de Alba G. como propuesta que se embebe de los principios del transfeminsimo español y se materializa en una obra poética ilustrada con el título Estoy más buena que Dios, editada en 2016 por La Novia de Nadie Ediciones. Este poemario de carácter autobiográfico transita por temas cotidianos del feminismo, las prácticas políticas autónomas y los movimientos sociales, por lo que se habita en los centros sociales autogestionados y nos hace partícipes de las contradicciones que se viven de manera encarnada. La frescura, el descaro, la ironía y el humor nos parecen realmente valiosos en la ópera prima de Alba G. Pero, seguramente, lo más reseñable es el ejercicio de reapropiación poética y resignificación constante que realiza de lo considerado como abyecto en el régimen de género hegemónico. Para poder disfrutar de las ilustraciones de María Rodríguez que acompañan cada poema, recomendamos que acudáis a la fuente original.

En la sección Llibres contamos con cuatro propuestas. Rosa María Medina Doménech reseña la obra Relaciones amorosas de las mujeres encarceladas, de Estibaliz de Miguel Calvo (2016). Estibaliz de Miguel Calvo reseña Love. A question for feminism in the Twenty-first Century, obra editada por Anna G. Jónasdóttir y Ann Ferguson (2014). Sergio Fernández Martínez reseña Cartografías del cuerpo. Biopolíticas de la ciencia y la tecnología, compilación de trabajos editada por Eulalia Pérez Sedeño y Esther Ortega Arjonilla (2014). Por último, Víctor Ballester reseña TransfeminismosEpistemes, fricciones y flujos, obra editada por Miriam Solá y Elena Urko (2013).

Para finalizar, el número 32 de Asparkía está ilustrado con ocho collages digitales de Ana Reguera, firmados bajo el seudónimo Señora Milton. El collage digital en manos de esta diseñadora gráfica consigue despedazar y deconstruir el simbolismo de la cultura dominante -heteronormativa, clasista, sexista, racista, etnocentrista, etc.- para recontextualizarlo y crear piezas detallistas que invitan a resituar la lectura de imágenes de la cultura popular acerca del género. El collage de Señora Milton se convierte en un arma política que representa y altera la realidad social al mismo tiempo que cuestiona y aporta matices con la superposición, que pone a dialogar elementos culturales que se contradicen, complementan y explican tales distorsiones encadenando signos de modo que el pensamiento se complejiza en cada detalle.

Sus collages presentan temas recurrentes en el debate feminista actual como el poliamor, la sexualidad bajo el régimen heteropatriarcal, la trata de personas, la explotación sexual, la cobertura mediática de la violencia de género, la islamofóbia, 
lo queer y la interseccionalidad, así como muchos otros conceptos y realidades complejas que la autora sintetiza en composiciones que entretejen propuestas artísticas bellas, arriesgadas y honestas. La ironía y el humor que desprenden sus ilustraciones facilitan la decodificación de un legado simbólico que estructura e impone un orden social jerárquico, violento y cruel. De hecho, las piezas beben de elementos visuales de la cultura popular producidos por y bajo el capitalismo neoliberal transnacional, recogidos en revistas, anuncios, folletos y envoltorios. Materiales que, bajo la lógica punk del DIY, permiten cuestionar y subvertir las narrativas dominantes.

La selección de collages realizada para la ocasión son trabajos realizados para la revista feminista digital Pikara, la organización CEAR Euskadi, la Fundación Atenea, la revista Harper's Bazaar España, el libro Barbarismos queer y otras esdrújulas (Platero, Rosón, y Ortega, 2017) y una pieza de su obra personal que hemos dedicado a la portada del número. Una pequeña muestra del universo simbólico de Señora Milton que nos deja con una sonrisa (o una mueca) y un flujo de reflexión que no acaba con el último detalle de cada propuesta ${ }^{6}$.

\section{Por una política de la diversión}

Los afectos están presentes en los movimientos sociales y el goce y el placer funcionan como argamasa del activismo (Jasper, 1998) y, de hecho, tienen un papel fundamental para poner en práctica estrategias de acción que subvierten los modelos hegemónicos de ciudadanía (desde los grupos de autoconsciencia, los espacios no mixtos o las prácticas de autogestión de la vida). En el desarrollo de las políticas prefigurativas (Maeckelbergh, 2011; Yates, 2014), es decir, en el establecimiento de relaciones que ya suponen un desafío al orden de género y que se entrevén los contornos del cambio social, el componente afectivo, lúdico, de experiencia vital, constituye el entramado en el que las diversiones, las prácticas diversas desde encarnaciones de género no hegemónicas -no sin contradicciones, tensiones y puntos de fuga- toman forma.

A lo largo de los contenidos de este número de Asparkía aparecen, se desgranan y se disfrutan algunos ejemplos de prácticas políticas, activistas, de creación artística, literarias, etc. que mantienen esa voluntad de cambio social a través de la resignificación, la reapropiación y la experimentación más allá de los límites de lo hegemónico. Por otra parte, y con la misma intensidad, los contenidos tratan de desgranar los mecanismos del poder, poner de manifiesto las constricciones de género e identificar las prácticas del poder, del legado simbólico, de las constricciones heteropatriarcales, clasistas y racistas:

El pensamiento queer que no apela a la normalización como sinónimo de legitimidad, de la misma forma que la noción de agencia no se identifica necesariamente con el uso de empoderamiento que el feminismo institucional incorpora, nos muestra también que frente al monopolio de la violencia existen

6 Para ver más, su porfolio se encuentra disponible en el siguiente enlace https://madamemilton. myportfolio.com/ 
frentes de resistencia que pueden entremezclar su agencia con una visión de activismo lúdico-crítico y anticapitalista, sin que esto deba entenderse de forma abstracta o superficial, dado que implica una autocrítica y una revisión reflexiva con respecto al papel de la resistencia queer frente a la mercantilización transnacional de los cuerpos y del consumismo global como ideología neoliberal (Villaplana, 2017: 22)

Las posibilidades del agenciamiento desde lo lúdico, lo placentero y lo bello, etc., tal y como expresa de manera magistral Villaplana en la cita de antes, son impensables siempre que incorporen la crítica aguda y fundamentada de la estructura de relaciones de género, siempre que no dejen de perseguir cambios fehacientes en la estructura de las relaciones de género tanto en el ámbito del poder, como la producción, el deseo y el simbolismo. Cambios que afecten, no solo a las personas y colectivos que por su ubicación específica en la estructura social tiene capacidad de hacerlo, sino que persiga transformaciones que afecten a las personas y colectivos en precareidad, entendida esta según Butler, como condición política inducida sobre algunas poblaciones que adolecen de falta de redes de apoyo sociales y económicas, y se encuentran más expuestas a los daños, la violencia y la muerte (Butler, 2014).

Con todas estas reflexiones todavía y por mucho tiempo en nuestras mentes, en la academia y en la calle, no podemos finalizar sin agradecer las aportaciones de todas las personas que han hecho posible este monográfico con sus contribuciones, revisiones, creaciones y posterior maquetación. Y, cómo no, a l'Institut Universitari d'Estudis Feministes $i$ de Gènere Purificación Escribano (IF) por hacer posible este número.

\section{REFERENCIAS BIBLIOGRÁFICAS}

Bourdieu, Pierre (2000): La dominación masculina, Barcelona: Anagrama.

ButLER, Judith (2002): Cuerpos que importan. Sobre los limites materiales y discursivos del «sexo», Barcelona: Paidós.

Butler, Judith (2007): El género en disputa, Madrid: Paidós.

ButLER, Judith (2009): «Precariedad, precaridad y políticas sexuales», AIBR. Revista de Antropología Iberoamericana, Vol.4, 3, pp. 321-336.

Butler, Judith y Athena Athanasiou (2013): Dispossession: The performative in the political, Cambridge: Polity Press.

Butler, Judith (2014): «Rethinking Vulnerability and Resistance», Madrid, June 2014, Recuperado de http://www.institutofranklin.net/sites/default/files/files/ Rethinking\%20Vulnerability\%20and\%20Resistance\%20Judith\%20Butler.pdf

DAvis, Angela Y. (2004): Mujeres, raza y clase, Móstoles: Ediciones Akal, 1981.

Connell, Raewyn (2006): «Glass Ceilings or Gendered Institutions? Mapping the Gender Regimes of Public Sector Worksites», Public Administration Review, Vol. $66, \mathrm{~N}^{\mathrm{o}} 6$, pp. 837-849.

Connell, Raewyn (2009): Gender. Second edition, Cambridge: Polity Press. 
Connell, Raewyn y Rebeca Pearse (2018): Género desde una perspectiva global, València: PUV (Traducción de Arantxa Grau i Muñoz y Almudena A. Navas Saurín).

Foucalut, Michel (1983): El discurso del poder, México: Folios Ediciones.

Foucalut, Michel (1978): Historia de la sexualidad. La voluntad de saber, Madrid: Siglo XXI. Foucalut, Michel (1978b): Microfísica del poder, Madrid: La Piqueta.

JASPER, James M. (1998): «The Emotions of Protest: Affective and Reactive Emotions In and Around Social Movements», Sociological Forum, Vol. 13, N 3, pp 397-424. JónAsDótTiR, Ana G. (1993): El poder del amor. ¿Le importa el sexo a la democracia? Madrid: Cátedra.

Mafckelbergh, Marianne (2011): «Doing is believing: Prefiguration as strategic practice in the alterglobalization movement», Social Movement Studies, Vol 10, $\mathrm{N}^{\mathrm{o}}$ 1, pp. 1-20.

McDowell, Linda (2000): Género, identidad y lugar, Madrid: Cátedra.

Mohanty, Chandra Talpade (1988): «Under Western Eyes: Feminist Scholarship and Colonial Discourses», Feminist Review, No 30, pp. 61-88.

Osborne, Raquel y Cristina Molina Petit (2008): "La evolución del concepto de género. Selección de textos de $\mathrm{S}$ de Beauvoir, K Millet, G Rubin y J Butler», Empiria: Revista de metodología de ciencias sociales, $\mathrm{N}^{\mathrm{0}} 15$, pp. 147-182.

Platero, R. Lucas; Rosón, María y Esther Ortega (eds.) (2017): Barbarismos queer y otras esdrújulas, Barcelona, Bellaterra.

Villaplana, Virginia (2017): «Agencia», en Platero, R. Lucas; Rosón, María y Esther ORTEGA (eds.): Barbarismos queer y otras esdrújulas, Barcelona: Bellaterra.

WitTig, Monique (2006): El pensamiento heterosexual y otros ensayos, Barcelona: Egales.

YATES, Luke (2014): «Rethinking Prefiguration: Alternatives, Micropolitics and Goals in Social Movements», Social Movement Studies, Vol. 14, Nº 1, pp. 1-21. 